\title{
Estimates of slope erosion intensity utilizing terrestrial laser scanning
}

\author{
BULAT USMANOV, OLEG YERMOLAEV \& ARTUR GAFUROV \\ Kazan Federal University, Department of the Landscape Ecology, Institute of Ecology and Geography, \\ 18 Kremlevskay St., Kazan, Russia, 420008 Kazan, Russia \\ busmanof@kpfu.ru, oyermol@gmail.com
}

\begin{abstract}
Despite the large variety of methods for estimating slope erosion intensity, it is still difficult to obtain accurate erosion rates. Therefore, our goal was to develop a method to provide accurate estimates of sheet and rill erosion intensities, and evaluate denudation quantities due to abrasion, landslides and talus processes using a high-precision laser scanning system (Trimble ${ }^{\circledR}$ GX). Differential maps between all stages of surveying and TIN-models were built directly on point clouds in "Trimble ${ }^{\circledR}$ RealWorks" software. Inspection and cross-section tools were used for detailed study of ground movements on the slope surface and the development of linear erosion forms. A new method for accurate estimates of the erosion has been developed using terrestrial laser scanning techniques. It makes it possible to assess the denudationaccumulation balance on erosive slopes, determine the dynamics of the volume of material moved on different parts of the slope in various surface runoff events, and identify spatial regularities forming rill washouts.
\end{abstract}

Key words erosion; denudation; abrasion; landslides; laser scanning; TIN-model; cloud of points

\section{INTRODUCTION}

Erosion on cultivated slopes is a major cause of anthropogenic soil degradation because naturaland human-induced erosion may substantially exceed natural recovery of soils. Therefore, significant effort has been dedicated to development of large sets of erosion measurement methods around the world. The problem of quantifying erosion intensity in various segments of temporary hydrographic networks remains open due to a large variety of surface runoff types induced by forces of snowmelt and rainwater. A large number of factors can influence erosion intensity and its spatial distribution, making observation of erosion intensity complicated. It is known that slopes develop sheet, rill and linear gully erosion with different corresponding velocities. Most observation methods are developed for gully erosion due to its good morphological reflection in relief and high flow velocity. However, none of the existing methods allows estimation of real rates of soil loss as a result of sheet and rill erosion. This is primarily due to the relatively low speed of soil losses, morphological fuzziness of erosion forms and the complexity of performing repeated observations on cultivated lands. Nevertheless, assessment of sheet and rill erosion intensities is a very important task since it adversely affects almost all cultivated lands and more than $2 / 3$ of the slopes that are dominant over extensive areas of forest-steppe and steppe zones of the Russian Plain.

Numerous research papers provide insight into intra- and inter-annual observation techniques for rill erosion (Gregory \& Walling, 1976; Zaslavskiy, 1983; Yermolaev, 2002; Golosov, 2006). The most common approaches include the method of runoff plots, linear erosion volume measurement, photogrammetric method, soil surface photographic profiling, tracer method and others. All these methods have their advantages and disadvantages, therefore researchers are generally very careful when making conclusions about eroded soil volumes and washout layers.

Recently, emergence of new surveying equipment (such as terrestrial laser scanners (TLS)) stimulated development of new methods suitable for soil erosion assessment. New capabilities allow remote evaluation of arable land with highest accuracy (fraction of a $\mathrm{mm}$ ) and, inaccessible to traditional techniques, density of data points (millions of measurement points). It is important to keep in mind that TLS techniques have a number of practical advantages: high data acquisition speed without precision loss; fully digitized model of an object; data acquisition independent of lighting conditions; access to remote and complex objects; full automation of the measurement process; measurements of geometric parameters using digital 3D-model; data storage in a digital form; multiple use of laser scanned results. 
Application of terrestrial laser scanning methods for soil erosion is in its early phase. TLS methods have been used for recording of soil erosion changes due to deforestation (Dąbek et al., 2014), fires (Staley et al., 2014) or rain storms (Bechet et al., 2014), and quantitative estimates of changes in river banks as a result of fluvial processes (O'Neal \& Pizzuto, 2010; Vaaja et al., 2011). TLS are equally efficient for monitoring and estimates of rill erosion and in collecting continuous data from deeply incised gullies (Perroy et al., 2010) or landslide processes (Dunning, 2009). However, no research publications are available which elucidate rill erosion on the slopes for periods of melting and shower runoff.

In general, data collection and subsequent pre-processing look as follows:

(a) provide an orientation for each of the scans via targets (Vaaja et al., 2011) or specific

distinction points (Afana et al., 2010);

(b) scanning with maximal accuracy;

(c) filtering out of background "noise" and artifact elimination (Vaaja et al., 2011);

(d) scan registering (Dunning, 2009).

A range of laser scanning systems and corresponding software are available, providing the opportunity to select the most effective package for processing the registered data: Surfer 8-10 (Bechet et al., 2014), Polyworks, I-Site Studio, Cyclone, Faro-Scene, JRC-3D- Reconstructor (Afana et al., 2010), Riegl Riscan PRO (Perroy et al., 2010), etc.

\section{STUDY AREA AND EXPERIMENT}

Our report focuses on the development and application of the TLS technique for quantitative estimates of slope erosion intensity. For our tests, we chose a bare plot on the left bank of the

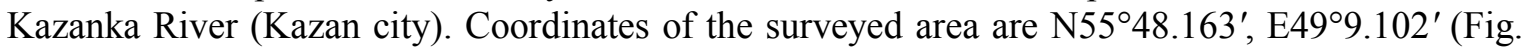
1), and slope characteristics: average inclination $31.5^{\circ}$, southwestern exposure, average slope length $-18 \mathrm{~m}$, relative excess $-9 \mathrm{~m}$, slope has a linear shape in cross-section. Plot size is 0.07 hectares. Below the soil layer the lithology is heavy deluvial-solifluction loams.

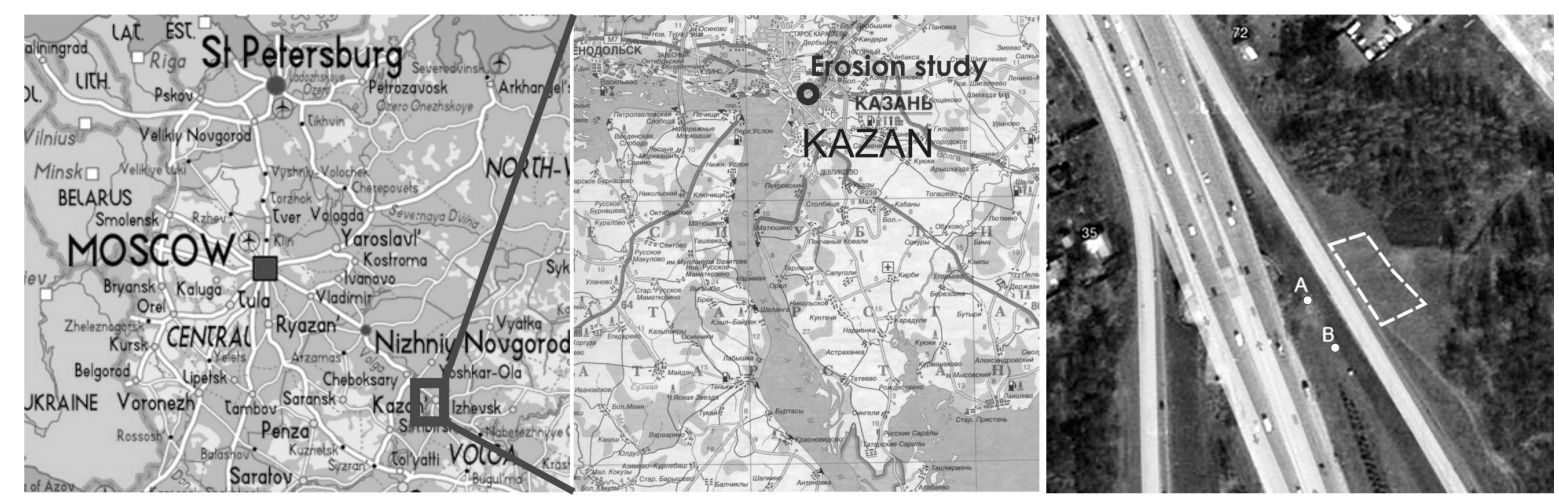

Fig. 1 Investigated area (A and B are the scanner position).

The site studied was chosen because of the exposure of rill complexes due to a lack of topsoil resulting from works related to slope improvements. The first scans were performed in April-June 2012 (Fig. 2(a)) followed by another scan in the fall of 2013. In the spring of 2013, erosion-control measures were implemented on the slope by Kazan municipal services, i.e. a geogrid covered with a layer of peat $(5-7 \mathrm{~cm})$ and seeded with grass. However, as a result of intense summer rain storms and a large layer of sediments in the fall (September-October) the slope rill erosion was fixed. Grass cover, a serious obstacle for laser scanning, also was washed away. Thus, observations of rill and sheet erosion development after snowmelt runoff (2012) and during rains in the autumn of 2013 were performed.

Erosion study with TLS began in 2012 and a small fragment of slope (C in Fig. 3) was scanned. In 2013, two plots (A and B in Fig. 3) without sod were selected for analysis, they are 

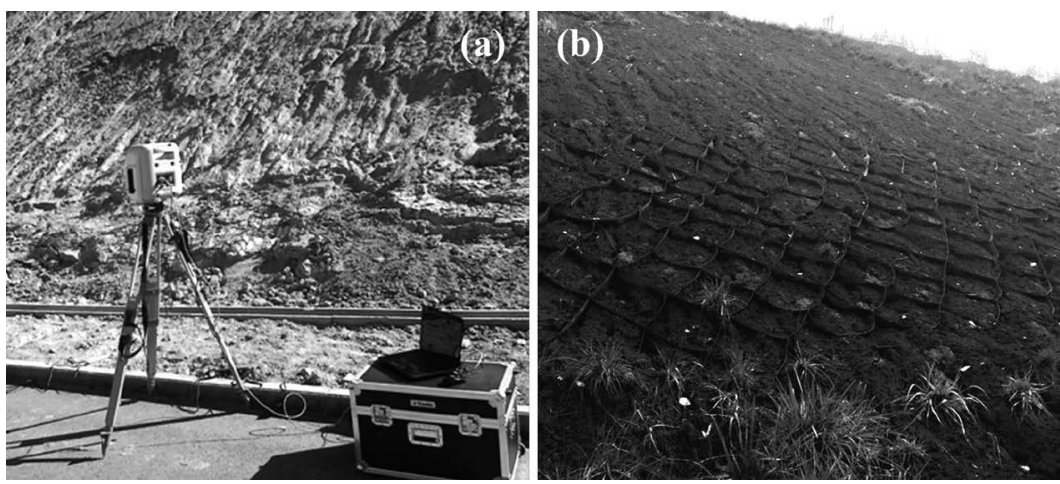

Fig. 2 Plot view in: (a) 2012 and (b) 2013.

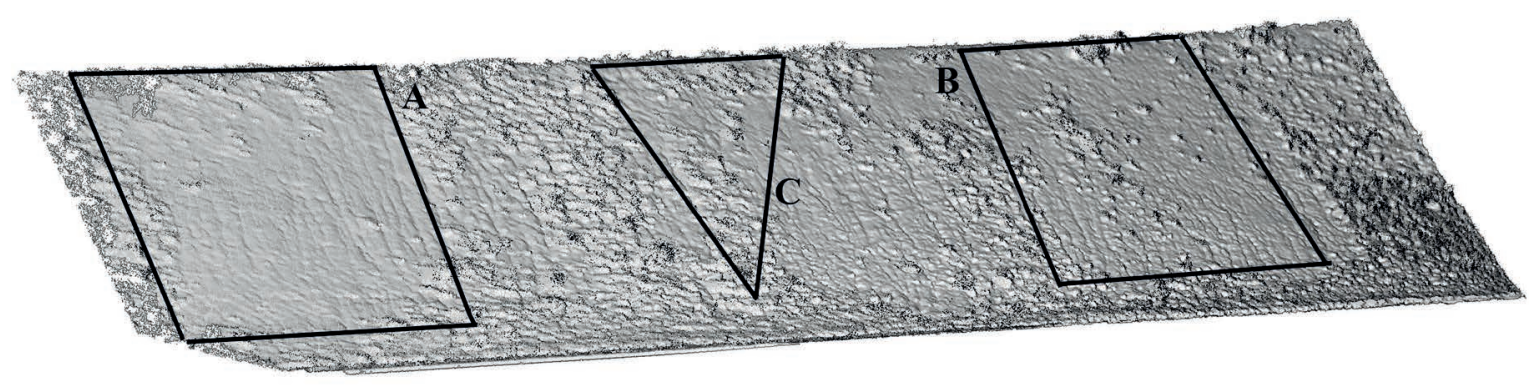

Fig. 3 Fragments of the experimental plot selected for further analysis: A and B - 2013; C - 2012.

visually different due to the intensity of erosion processes - on the left the rill network is denser and morphology is better expressed. On some sections of the plot slope, runoff fully washed away the soil layer and exposed the geogrid (Fig. 2(b)).

\section{ORGANIZATION OF OBSERVATIONS}

The studied slope was scanned by a Trimble ${ }^{\circledR}$ GX scanner with a Trimble ${ }^{\circledR}$ PointScape software interface. This equipment allows acquisition of data from a distance of up to $350 \mathrm{~m}$ with the measurement speed of up to 5000 points per second and accuracy of 1.4-6.5 mm (depending on the distance); the resolution at $100 \mathrm{~m}$ was determined to be $3 \mathrm{~mm}$ with a small angular size of a laser spot. Used parameters allowed accurate reconstruction of a 3-D model of the studied object.

Environmental conditions have a negative effect on scanning measurements, e.g. distance, horizontal and vertical angles, intensity and real colour of the surface object. This is especially true for surface layers due to changes in air density, movements and fluctuation of air masses that lead to reduction of light spreading rate and to refraction phenomena, as well as a decrease of reflected radiation intensity and incorrect colour rendering ("haze" effect).

Surface brightness can also affect the scanning speed. Results of the observations demonstrated that brightly illuminated objects require more time to complete the measurements. It is necessary to take all technical parameters into account to ensure scanning accuracy and record sheet and rill erosion.

Considering the above mentioned factors, we found the following steps are important for accurate surveying of erosion slope:

(a) Systematic surveying over several years, especially after snowmelt runoff and intense rainfall;

(b) Rainfall data collection at the time of scanning;

(c) Rescanning with the same light conditions;

(d) Putting laser scanner on the opposite side of a studied area at elevation that provides maximum coverage in one scan and minimizes "shadows" in the point cloud;

(e) Recommend distance to surveyed area shall not exceed $100 \mathrm{~m}$, because scanning accuracy decreases with a distance increase; 
(f) Rigid mounting of scanner horizontally and vertically to minimize errors during rescanning;

(g) Installation of surveying targets on static structures (fences, lighting poles, buildings, etc.);

(h) $360^{\circ}$ panning with scanner to simplify matching of point cloud with scanning surfaces;

(i) Prescanning the area around the scanner to register the point cloud by object if software does not recognize special target;

(j) Scanning with appropriate resolution depending on erosion form sizes, bearing in mind that scanning with high resolution takes more time;

(k) Before scanning, adjust gauge scanner with settings to: point density $-15 \mathrm{~mm}$ on $25 \mathrm{~m}$ distance; 4 laser shots per point; survey mode - fast (colour).

\section{DATA PROCESSING}

The processing of output data was accomplished with Trimble ${ }^{\circledR}$ RealWorks 6.5 software. The software allowed registering and editing $3 \mathrm{D}$ point clouds, as well as performing various calculations.

For binding survey results acquired on different dates, target-based registration was carried out in a semi-automatic mode. Then, differential maps were reconstructed and superimposed on a studied slope. Differences between point positions were identified by a coloured scale (Fig. 4).
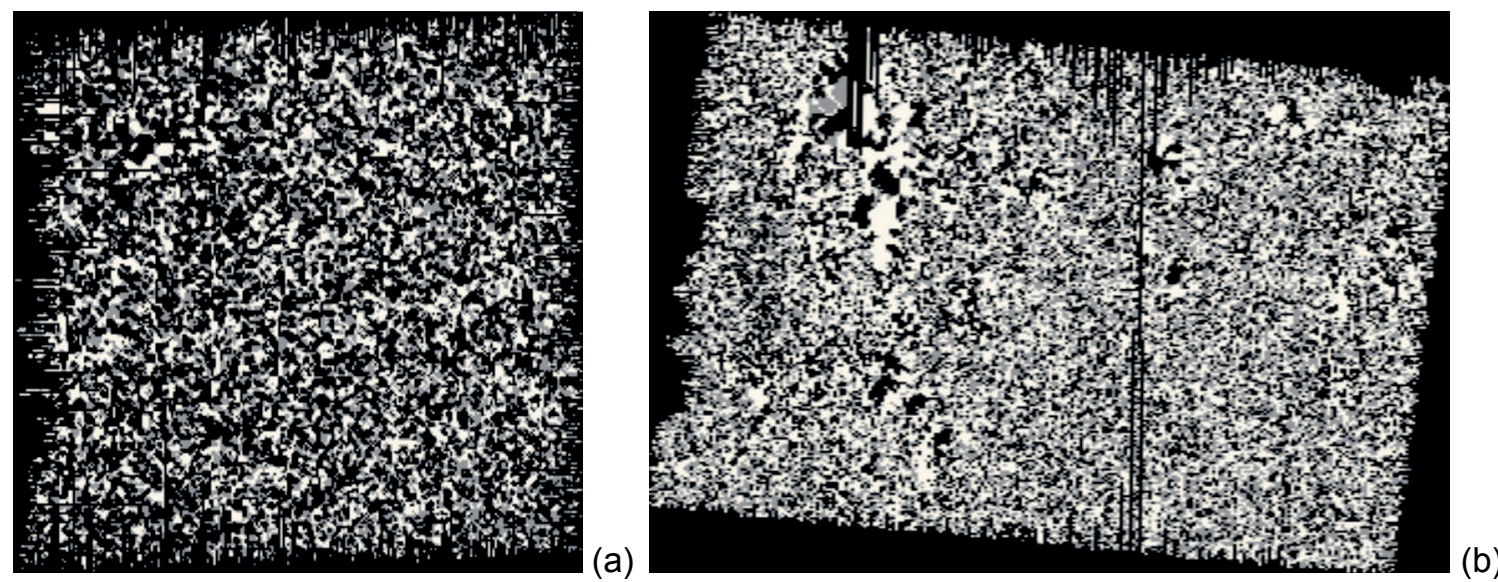

Fig. 4 Example of differential maps in the middle section of the slope: (a) plot A, (b) plot B. White accumulation, black - erosion, grey - absence of changes within the scanner accuracy $( \pm 2.5 \mathrm{~mm})$.

At the next step, changes on the slope were quantified as a result of erosion and accumulation processes, and the volume of washout and accumulation after each runoff event were calculated. For clarity, maps of differences were superimposed on the TIN-model created on the base of the point cloud. Moreover, we determined quantitative characteristics of erosion and accumulation processes in the upper, middle and lower parts of the slope. For this, we calculated decreases and increases $\left(V_{-}, V_{+}, \mathrm{m}^{3}\right)$ of ground volume in the studied part of a slope via the "Volume calculation" tool. Then, using plot area $(S)$, we calculated erosion $(i+)$, accumulation $(i-)$ layers and predominant process $(\Delta i)$, as well as the erosion-accumulation volume $\left(E, \mathrm{~m}^{3} / \mathrm{ha}\right)$ :

$$
i-=\frac{V_{-}}{S}, \mathrm{~mm}, i+=\frac{V_{+}}{S}, \mathrm{~mm}, \Delta i=\frac{V_{+}-V_{-}}{S}, \mathrm{~mm}, E=\frac{V_{+}-V_{-}}{S} 10000, \mathrm{~m}^{3} / \mathrm{ha}
$$

\section{RESULTS}

The first series of observations were conducted in 2012 after snowmelt and the first two rainstorms formed the runoff. At that time, the slope was entirely exposed and was not covered with any soil, but with heavy deluvial-solifluction loam with a density $1.6 \mathrm{~g} / \mathrm{cm}^{3}$. After the snowmelt runoff, due to the steepness, high water content and composition of the rocks, an earthflow formed across the front of the middle and lower parts of the slope. Their appearance is connected with solifluction 
and landslides. The occurrence of these processes was observed during snowmelt in March (6-9 March) 2014. The thickness of snow cover at this time was $33-53 \mathrm{~cm}$, water reserve in the snow (at a density of $0.3 \mathrm{~g} / \mathrm{cm}^{3}$ ) ranged from $120 \mathrm{~mm}$ in the upper and middle to $168 \mathrm{~mm}$ - in the lower part of the slope. However, at high intensity of snowmelt (snow melted completely in 3-4 days) visible flows did not form. Snowmelt occurred due to radiative evaporation in the daytime (cloudless weather established), large effective radiation from the surface at night and its own long-wave radiation of snow deep layers. As a result, during 3-4 days the slope at the upper and the middle parts was completely free from snow. At the same time, the soil is thawed only by $0.5-$ $1.5 \mathrm{~cm}$. Water coming from the snow greatly moistened the ground layer, consequently it acquired flow properties at high inclines of slope. As a result, there was widespread development of solifluction and sliding processes.

Later, almost all constituents of the landslides were washed out by stormwater runoff in the form of rill erosion. A morphologically well-defined dense network of streams formed there. The maximum width of the streams was $15-20 \mathrm{~cm}$, and depth $8-10 \mathrm{~cm}$. Analysis of the results (Table 1) indicates a high intensity of exogenous processes. At the upper part of the slope, accumulation is dominant ( $3.5 \mathrm{~mm}$ or $57 \mathrm{t} / \mathrm{ha}$ ); in the middle part denudation is observed $(4.1 \mathrm{~mm}$ or $66.1 \mathrm{t} / \mathrm{ha})$, as well as at the lower part $(4.3 \mathrm{~mm}$ or $68.4 \mathrm{t} / \mathrm{ha}$ ). In general, during the observation period (AprilJune 2012) the denudation layer across the slope was $0.6 \mathrm{~mm}$ (or $10.4 \mathrm{t} / \mathrm{ha}$ ). Taking into account the fact that steepness of the observed areas is significantly higher than that of arable land areas, these values are close to averages previously obtained for agricultural soils and forest-steppe zone of the Russian Plain (Yermolaev, 2013, 2014).

Table 1 Results of data processing April-June 2012 (Plot C).

\begin{tabular}{lllllllll}
\hline Slope part & $\begin{array}{l}S \\
\left(\mathrm{~m}^{2}\right)\end{array}$ & $\begin{array}{l}V_{-} \\
\left(\mathrm{m}^{3}\right)\end{array}$ & $\begin{array}{l}V_{+} \\
\left(\mathrm{m}^{3}\right)\end{array}$ & $\begin{array}{l}V_{+-} V_{-} \\
\left(\mathrm{m}^{3}\right)\end{array}$ & $\begin{array}{l}i- \\
(\mathrm{mm})\end{array}$ & $\begin{array}{l}i+ \\
(\mathrm{mm})\end{array}$ & $\begin{array}{l}\Delta i \\
(\mathrm{~mm})\end{array}$ & $\begin{array}{l}E \\
\left(\mathrm{~m}^{3} / \mathrm{ha}^{2}\right.\end{array}$ \\
\hline C, upper & 18.3 & 0.041 & 0.106 & +0.065 & 2.2 & 5.7 & +3.5 & +35.5 \\
C, middle & 14.0 & 0.080 & 0.022 & -0.058 & 5.7 & 1.6 & -4.1 & -41.4 \\
C, lower & 7.7 & 0.040 & 0.007 & -0.033 & 5.2 & 0.9 & -4.3 & -42.9 \\
Total, plot $C$ & 40 & 0.161 & 0.135 & -0.026 & 4.0 & 3.4 & -0.6 & -6.5 \\
\hline
\end{tabular}

$\mathrm{S}$, plot area; $\mathrm{V}_{-}$decreases of ground volume; $\mathrm{V}_{+}$, increases of ground volume; i+, accumulation layer; $\mathrm{i}_{-}$, erosion layer; $\Delta \mathrm{i}$, predominant process; $\mathrm{E}$, erosion-accumulation volume.

The second series of observations were carried out in the autumn of 2013. As already noted, in the summer the slope was covered with a layer of peat that was artificially compacted for lawn planting. The average density of the soil layer was $0.8 \mathrm{~g} / \mathrm{cm}^{3}$. During the summer heavy rains, a system of rill erosion formed on a slope, but erosion assessment using TLS was not carried out due to a large grass cover impeding the measurements. Recognizing the obvious fact that the main erosion on slopes was a result of heavy rains, we set the goal of its evaluation during the autumn rains. This aspect of fluvial geomorphology is poorly studied since it is assumed that slope runoff is minimal in the autumn due to low rainfall intensity, i.e. no erosion without effective precipitation. However, our research showed opposite results. One needs to take into account not only characteristics such as rainfall intensity, but also other parameters: level of soil moisture, vegetation cover, slope morphometry, etc.

During the autumn observation period (September-October 2013) $124 \mathrm{~mm}$ of precipitation was recorded. The soil layer was already saturated by September rain. Apparently, this factor was the main contributor to slope runoff formation during low intensity rainfall. The slope was scanned from two stations (Fig. 1) on 4 and 18 October 2013, after several rains with large precipitation layer. In particular, the first survey (4 Oct. 2013) was performed after three rainfalls amounting to a total of more than $70 \mathrm{~mm}$; the second (18 Oct. 2013) was after an $18.1 \mathrm{~mm}$ rainfall.

The erosion and accumulation results are shown in Table 2. On the right (B) plot in all parts of the slope weak accumulation is dominant $(0.9 \mathrm{~mm}(\approx 7 \mathrm{t} / \mathrm{ha}))$, and its values lie within scanner accuracy limits. On the left (A) plot, in contrast, erosion prevails, the middle layer of the denudation was $2.4 \mathrm{~mm}$ or $19.4 \mathrm{t} / \mathrm{ha}$. 
Table 2 Results of data processing 4 and 18 October 2013 (A and B plots).

\begin{tabular}{lllllllll}
\hline Slope part & $\begin{array}{l}S \\
\left(\mathrm{~m}^{2}\right)\end{array}$ & $\begin{array}{l}V_{-} \\
\left(\mathrm{m}^{3}\right)\end{array}$ & $\begin{array}{l}V_{+} \\
\left(\mathrm{m}^{3}\right)\end{array}$ & $\begin{array}{l}V_{+}-V_{-} \\
\left(\mathrm{m}^{3}\right)\end{array}$ & $\begin{array}{l}i- \\
(\mathrm{mm})\end{array}$ & $\begin{array}{l}i+ \\
(\mathrm{mm})\end{array}$ & $\begin{array}{l}\Delta i \\
(\mathrm{~mm})\end{array}$ & $\begin{array}{l}E \\
\left(\mathrm{~m}^{3} / \mathrm{ha}\right)\end{array}$ \\
\hline A, upper & 23.92 & 0.13 & 0.07 & -0.06 & 5.4 & 2.9 & -2.5 & -25.08 \\
A, middle & 26.07 & 0.12 & 0.05 & -0.07 & 4.6 & 1.9 & -2.7 & -26.85 \\
A, lower & 24.27 & 0.11 & 0.06 & -0.05 & 4.5 & 2.4 & -2.1 & -20.60 \\
Total, Plot $A$ & 74.26 & 0.36 & 0.18 & -0.18 & 4.8 & 2.4 & -2.4 & -24.23 \\
\hline B, upper & 39.24 & 0.09 & 0.12 & +0.03 & 2.3 & 3.0 & +0.7 & +7.65 \\
B, middle & 46.37 & 0.11 & 0.14 & +0.03 & 2.4 & 3.0 & +0.6 & +6.47 \\
B, lower & 41.06 & 0.09 & 0.14 & +0.05 & 2.2 & 3.4 & +0.8 & +12.18 \\
Total, Plot B & 126.67 & 0.29 & 0.4 & +0.11 & 2.3 & 3.2 & +0.9 & +8.68 \\
\hline
\end{tabular}

$\mathrm{S}$, plot area; $\mathrm{V}_{-}$, decreases of ground volume; $\mathrm{V}_{+}$, increases of ground volume; i+, accumulation layer; i-, erosion layer; $\Delta \mathrm{i}$, predominant process; $\mathrm{E}$, erosion-accumulation volume.

Accumulation on Plot B occurs most likely due to the undulating shape of the transverse profile, weak lateral water inflow from the general watershed, and sod cover fragments in the upper and middle part of the slope preventing runoff formation. Visible changes in the rill system were not detected. Soil and ground mass movement occurs mainly through their dislodging and colmatation. These processes are clearly visible on the side walls of rills.

On Plot A denudation is widely developed; it mostly occurs due to the fact that it has an almost perfect straight shape in cross-section. As opposed to Plot B, it is more exposed and is subject to lateral inflow of water coming from the top of the general watershed; it also has an overall inclination towards the Kazanka River $\left(\approx 3^{\circ}\right)$.

\section{CONCLUSION}

A new method for quantitative evaluation of rill and sheet erosion intensity on slopes has been developed using terrestrial laser scanning techniques. This method utilizes high precision surveying techniques and allows recording of various types of erosion. Consequently, it is possible to assess the denudation-accumulative balance on erosive slopes, determine the dynamics of the volume of material moved in different slope parts in various events of surface runoff, and identify spatial regularities forming rill washouts. Furthermore, this method allows accurate estimates of the erosion taking into account the integrated effect of all exogenous processes. The TLS method can play an important role in determining the regularities of gully form development. As with any other methods, TLS method has its own limitations: scans with high accuracy are only possible on completely exposed surfaces of soils and ground; difficulties associated with repeating observations on arable land (benchmark installation and preservation); registration requirements for the scanning conditions; and, sometimes, excessively high scan density.

Acknowledgements This work was funded by RFBR-RGS (project 13-05-41126), RFBR (project 14-05-00503).

\section{REFERENCES}

Afana, A., Solé-Benet, A. \& Pérez, J.L. (2010) Determination of Soil Erosion Using Laser Scanners. 19-th World Congress of Soil Science, Soil Solutions for a Changing World, 1 - 6 August 2010, Brisbane, Australia. Published on DVD, $39-42$.

Bechet, J., et al. (2014) Technical Note: Erosion processes in black-marls at the millimetre scale, the input of an analogical model. Hydrol. Earth Syst. Sci. Discuss., 11, 2263-2275 (this discussion paper is under review).

Dabek, P., Zmuda, R., Ćmielewski, B. \& Szczepański, J. (2014) Analysis of water erosion processes using terrestrial laser scanning. Acta Geodynamica et Geomaterialia. 11(1), 45-52.

Dunning, S.A., Massey, C.I. \& Rosser, N.J. (2009) Structural and geomorphological features of landslides in the Bhutan Himalaya derived from Terrestrial Laser Scanning. Geomorphology 103(1), 17-29.

Golosov, V.N. (2006) Erosion-accumulative processes in river basins of cultivated plains. Moscow: GEOS (in Russian).

Gregory, K.Y. \& Walling, D.E. (1976) Drainage basin form and process: a geomorphological approach. Edward Arnold Ltd.

O'Neal, M.A. \& Pizzuto, J.E. (2011) The rates and spatial patterns of annual riverbank erosion revealed through terrestrial laserscanner surveys of the South River, Virginia. Earth Surface Processes and Landforms. 36(5), 695-701. 
Perroy, R.L., et al. (2010) Comparison of gully erosion estimates using airborne and ground-based LiDAR on Santa Cruz Island, California. Geomorphology. 118(3-4), 288-300

Staley, D.M., Wasklewicz, T.A. \& Kean, J.W. (2014) Characterizing the primary material sources and dominant erosional processes for post-fire debris-flow initiation in a headwater basin using multi-temporal terrestrial laser scanning data. Geomorphology.

Vaaja, M., et al. (2011) Mapping topography changes and elevation accuracies using a mobile laser scanner. Remote Sensing. 3(3), 587-600.

Yermolaev, O.P. (2002) Erosion in basin geosystems. Unipress KGU, Kazan (in Russian).

Yermolaev, O.P. (2013) Assessment of the suspended sediment yield in the rivers' basin of the Russian Plain. World Applied Sciences Journal. 27 (5), 626-631.

Yermolaev, O.P. (2014) Erosion processes of the forest and forest-steppe zones in the Eastern Part of the Russian Plain. World Applied Sciences Journal. 29 (3), 453-459.

Zaslavsky, M.N. (1983) Eroziovedenie. Moscow: Vysshaya Shkola (in Russian) 